\title{
THE SMALLEST POSITIVE INTEGER THAT IS SOLUTION OF A PROPORTIONALLY MODULAR DIOPHANTINE INEQUALITY
}

\author{
J. C. Rosales AND P. VASCO
}

Abstract. A proportionally modular Diophantine inequality is an expression of the form $a x \bmod b \leqslant$ $c x$, where $a, b$ and $c$ are positive integers. In this paper we present an algorithm that allows us to calculate the smallest positive integer that is solution of an inequality of this type. We also obtain an algorithm that computes the Frobenius number and the number of gaps of a numerical semigroup generated by three positive integers.

Mathematics subject classification (2000): 20M14, $13 \mathrm{H} 10$.

Key words and phrases: numerical semigroup,Diophantine inequality, multiplicity, Frobenius number, gaps.

\section{REFERENCES}

[1] V. BaruCCI, D. E. DobBs AND M. FontanA, Maximality Properties in Numerical Semigroups and Applications to One-Dimensional Analytically Irreducible Local Domains, Memoirs of the Amer. Math. Soc. 598 (1997).

[2] S. M. Johnson, A linear Diophantine problem, Can. J. Math. 12 (1960), 390-398.

[3] J. D. LIPSON, Elements of algebra and algebraic computing, Addison-Wesley, 1981.

[4] J. L. RAMÍREZ Alfonsín, The diophantine Frobenius problem, Oxford Univ. Press, 2005.

[5] Ö. J. RöDSETH, On a linear Diophantine problem of Frobenius, J. Reine Angew. Math. 301 (1978), $171-178$.

[6] J. C. Rosales AND P. A. GARCíA-SÁncheZ, Finitely generated commutative monoids, Nova Science Publishers, New York, 1999.

[7] J. C. Rosales AND P. A. GARCÍA-SÁnCHEZ, Numerical semigroups with embedding dimension three, Archiv Math (Basel) 83 (2004), 488-496.

[8] J.C. Rosales, P. A. García-SÁncheZ, J. I. García-García And J. M. URBano-Blanco, Proportionally modular Diophantine inequalities, J. Number Theory 103 (2003), 281-294.

[9] J. C. Rosales, and J. M. Urbano-Blanco, Proportionally modular Diophantine inequalities and full semigroups, Semigroup Forum 72 (2006), 362-374.

[10] J. J. SYLVESTER, Excursus on rational fractions and partitions, Amer J. Math. 5 (1882), 119-136.

[11] J. J. SYLVESTER, Mathematical questions with their solutions, Educational Times 41 (1884), 21. 